Kondakow: Ueb.d.Verh.d.Tetramethylendibromidsetc. 293

\title{
Ueber das Verhalten des Tetramethylendibromids zu alkoholischer Kalilauge;
}

von

Iwan Kondakow.

(Mittheilung aus dem pharmaceutischen Institut zu Jurjew.)

In einem meiner früheren Referate ${ }^{1}$ ) hatte ich auf einige Mängel des Baey er - Thi ele'schen ${ }^{2}$ ) Verfahrens zur Darstellung von Tetramethyläthylen hingewiesen und mich dahin ausgesprochen, „dass es augenscheinlich hinter dem Pawlow'schen zurücksteht." Eine Controllprüfung dieses letzteren Verfahrens jedoch überzeugte mich davon, dass es für den erwähnten

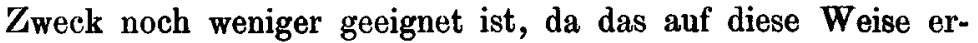
haltene Tetramethyläthylen stets eine, wenn auch geringe Beimengung von Amylen enthält, was darauf zurïckzuführen ist, dass das eine von den Ausgangsmaterialien - das Isobutyrilchlorid - eine, allerdings unbedeutende Beimengung von Propionylchlorid ${ }^{3}$ ) enthält. Die erwähnten Ursachen veranlassten mich, nach einem besseren Darstellungsverfahren des Tetramethyläthylens $\mathrm{zu}$ suchen und bei dieser Gelegenheit auch die Couturier'sche ${ }^{4}$ ) Methode zu erproben, obwohl man a priori auch nur wenig Vertrauen in die Tauglichkeit dieses Verfahrens setzen konnte. Ungeachtet dessen hielt ich es doch für unerlässlich, diese Methode zu untersuchen, und zwar hauptsächlich schon deswegen, weil die Bildung des Tetramethyläthylens durch Einwirkung alkoholischer Kalilauge auf Dibromtetramethyläthylen ${ }^{4}$ ), soviel mir bekannt ist, fast den einzigen

1) Dies. Journ. [2] 54, 429.

) Ber. 26, 2563 u. 27, 454 .

8) Es ist deswegen kaum anzunebmen, dass irgend einer von den Untersuchenden ein von dieser Verunreinigung freies Isobutyrylchlorid unter den Händen gehabt haben sollte.

4) Natriumphenylat reagirt, wie es scheint, gensu ebenso mit diesem Dibromid, wie aus den soeben im Druck erschienenen Untersuchungen 
294 Kondakow: Ueb.d.Verh.d. Tetramethylendibromidsetc.

Fall darstellt, der weder in der aromatischen noch in der aliphatischen Reihe Analogien aufweist. $\left.{ }^{1}\right)$

Das Tetramethyläthylen wurde, nach den Angaben Couturier's, durch Zerlegen des nach Ba eyer-Thiele bereiteten Pinacondibromids vermittelst alkoholischer Kalilauge dargestellt. Das rohe, auf diese Weise erhaltene Reactionsprodukt bildet, nachdem es behufs Entfernung des anhängenden Alkohols mit Wasser gewaschen und über Chlorcalcium getrocknet worden war, eine durchsichtige, sehr bewegliche Flüssigkeit, die leichter als Wasser ist und deren Geruch eher an den der Kohlenwasserstoffe der Allenreihe und des Aethylvaleryläthers als an den des Tetramethyläthylens erinnert. Diese Substanz siedet unbeständig, obgleich Couturier behauptet, dass ,il passe très exactement à $73^{\circ}-74^{\circ}$ : c'est le point d'ebullition du tétraméthyléthylène."

Bei der fractionirten Destillation unter einem Druck ron $753 \mathrm{Mm}$. ist das Rohprodukt in folgende Fractionen zerlegt worden: $1.69^{\circ}-74^{\circ} ; 2.74^{\circ}-130^{\circ}-$ sehr wenig; $3.130^{\circ}-140^{\circ}$ - ziemlich wenig, und 4. ein Rückstand, der unter vermindertem Druck destillirt wurde, da er sich bei der Destillation unter gewöhnlichem Druck merklich veränderte. Als die Temperatur bei der Destillation des Rückstandes unter einem Druck von $18 \mathrm{Mm}$. auf $60^{\circ}$ gestiegen war, begannen sich in der gekühlten Vorlage nadelförmige Krystalle auszuscheiden. In diesem Momente wurde die Destillation unterbrochen, worauf die ganze im Destillirkolben zuritickgebliebene Substanzmenge zu einer Krystallmasse erstarrte. Aus 115,0 Grm. Dibromid wurden ca. 6,0 Grm. Krystalle erhalten.

Zur Untersuchung der aus dieser Reaction resultirenden Substanz übergehend, muss ich vorausschicken, dass die Haupt-

von W. Solonina*) zu ersehen ist. Der Autor hat in seinem Referate aber der Untersuchungen von Couturier nicht Erwähnung gethan und auf das Abnorme bei der Bildung des Tetramethylithylens aus seinem Dibromide durch Einwirkung von Natriumphenylat nicht hingewiesen.

*) Journ. russ. phys.-chem. Ges. 30, 832.

1) Ein ahnnlicher Fall ist in der hydroaromatischen Reihe bekanut. Aus den Untersuchungen Wallach's (Ann. Chem. 253, 254) wissen wir, dass das Pinoldibromid unter dem Einfluss von alkoholischer Kalilauge 2 Atome Brom verliert. 
aufmerksamkeit auf die erste Fraction verwendet wurde. Diese Fraction - etwa zwei Drittel des ganzen Reactionsproduktes - wurde, nachdem durch die Beilstein'sche Reaction die Abwesenheit von Brom constatirt worden war, über Natrium destillirt, und wurde die Destillation so lange wiederholt, bis das Natrium aufhörte eine braunrothe Färbung anzunehmen. ${ }^{1}$ ) Der Kohlenwasserstoff siedet nach dieser Bearbeitung bei $69^{\circ}$ bis $74^{\circ}$ und $750 \mathrm{Mm}$.; sein Geruch ähnelt dem der Kohlenwasserstoffe der Allenreihe; er brennt mit russender Flamme, reagirt unter starkem Zischen mit Brom und entfärbt sofort Permanganatlösung. Sein spec. Gewicht $=0,7350$ bei $0^{\circ}, 0,7077$ bei $18^{\circ}$.

Das spec. Gew. des'Tetramethyläthylens beträgtnach $P$ a u low bei $0^{\circ}=0,712$, des Diisopropenyls nach Couturier . . . . . . bei $0^{0}=0,7565$.

Die Lichtbrechungsbestimmung des Kohlenwasserstoffs im Pulfrich'schen Apparate in der Natriumflamme ergab Folgendes:

bei $18^{\circ}=49^{\circ} 31^{\prime}$, d. h. Index $=1,4248$, woraus sich ergiebt:

Molekularrefraction . . . . . . . . . . $=29,32$.

Berechnet für $\mathrm{C}_{6} \mathrm{H}_{12}$ mit einer doppelten Bind. $=29,40$.

Zur weiteren Bestätigung des ungesättigten Charakters dieses Kohlenwasserstoffs wurde sein Verhalten zu Brom und zu den Halogenwasserstoffsäuren näher untersucht.

Um festzustellen, wie viel Brom addirt wird, wurden mehrere Versuche angestellt. Das erste Mal wurde die Reaction in ätherischer Lösung bei einer Temperatur von $-10^{\circ}$ ausgeführt. Die hierbei erbaltene krystallinische Verbindung hat einen ätzenden Geruch und enthält $66,21 \% \mathrm{Br}$, da 0,1631 Grm. Substanz 0,2541 Grm. AgBr gaben. Diese Krystalle lösen sich bei der Bearbeitung mit Petroläther fast vollständig auf, mit Ausnahme eines kleinen Rückstandes, welcher sich weder in Aether noch in Alkohol und Benzol löst. Der lösliche Theil scheidet sich aus seiner Petrolätherlösung in Form nadelförmiger Krystalle aus, welche den Krystallen

1) Wie es scheint, bildet sich irgend eine Natriumverbindung. Aehnliche Natriumverbindungen bilden sich auch bei der Destillation der Aethylenkohlenwasserstoffe mit Wasser. Bei der Zersetzung derartiger Verbindungen bilden sich tertiäre Alkohole (Trimethyläthylen und Tetramethyläthylen). 
296 Kondakow: Ueb.d.Verh.d.Tetramethylendibromidsete.

des Tetramethyläthylendibromids ähnlich sind. Sie schmelzen unbeständig bei $133^{\circ}-137^{\circ}$.

Die Brombestimmung ergab Folgendes:

0,1465 Grm. Substanz gaben $0,2248 \mathrm{AgBr}=65,25 \% \mathrm{Br}$.

Berechnet für $\mathrm{C}_{6} \mathrm{H}_{12} \mathrm{Br}_{2}$ : $\quad=65,56, \mathrm{Br}$.

Diese krystallinische Bromverbindung verändert sich leicht beim Aufbewahren sogar in zugeschmolzenem Röhrchen und nimmt zunächst eine gelbe, später eine dunkle Färbung an.

Nach 6monatlicher Aufbewahrung hatte sich diese Verbindung stark verändert und gab bei der Brombestimmung folgendes Resultat:

0,0593 Grm. Substanz gaben 0,0680 Grm. $\mathrm{AgBr}=48,48 \% \mathrm{Br}$.

$$
\text { Berechnet für } \mathrm{C}_{6} \mathrm{H}_{4} \mathrm{Br} \text { : } \quad=49,09, \mathrm{Br} \text {. }
$$

Beim zweiten Versuche wurde die Addition des Broms in Petrolätherlösung ausgeführt, wobei wiederum 2 Verbindungen erhalten wurden - eine lösliche und eine unlösliche.

Die Brombestimmung in den löslichen Krystallen ergab:

0,1415 Grm. Substanz gaben 0,2070 Grm. $\mathrm{AgBr}=62,23 \% \mathrm{Br}$.

Berechnet für $\mathrm{C}_{6} \mathrm{H}_{12} \mathrm{Br}_{2}$ : $\quad=65,56, \mathrm{Br}$. ergab:

Die Brombestimmung in der schwer löslichen Substanz

0,1415 Grm. Substanz gaben 0,2528 AgBr $=71,90 \% \mathrm{Br}$.

$$
\text { Berechnet für } \mathrm{C}_{8} \mathrm{H}_{10} \mathrm{Br}_{4} \text { : } \quad=79,60 " \mathrm{Br} \text {. }
$$

Der ungesättigte Charakter des Kohlenwasserstoffs documentirt sich auch, wie schon oben erwähnt, sehr deutlich durch die Eigenschaft, sich bei gewöhnlicher Temperatur mit Halogenwasserstoffsäure zu vereinigen. So vereinigt sich z. B. der Kohlenwasserstoff mit Chlorwasserstoffsäure vom spec. Gew. 1,19 bei gewöhnlicher Temperatur und giebt ein Chlorprodukt, welches bei $115^{\circ}-190^{\circ}$ unter Chlorwasserstoffausscheidung übergeht. Dieses Chlorprodukt addirt, sogar bei der Bearbeitung mit bei $-20^{\circ}$ gesättigter Säure im zugeschmolzenen Rohre bei $160^{\circ}$, keinen Chlorwasserstoff mehr. Bei der Destillation dieses Chlorprodukts im luftverdünnten Raume ist keine Zersetzung zu bemerken, und gegen das Ende der Destillation setzen sich am Kolbenhalse sternförmige Krystalle an, die den Schneekrystallen ähneln. Da dieses Chlorprodukt, welches augenscheinlich aus einem Gemisch von Chlorverbin- 
dungen bestand, weder durch fractionirte Destillation, noch durch Gefrierenlassen in seine Bestandtheile zerlegt werden konnte, so wurde es zunächst bei gewöhnlicher Temperatur im zugeschmolzenen Rohre mit Wasser bearbeitet. Hierbei löste sich nach Verlauf zweier Wochen nur ein Theil auf, welcher aus tertiärem Hexylchlorid bestand, da aus der sauern, wässrigen Lösung Dimethylisopropylcarbinol mit dem Siedepunkt $116^{\circ}-117^{\circ}$ ausgeschieden wurde. Der bei dieser Bearbeitung ungelöst zurückgebliebene Theil des Chlorprodukts wurde unter denselben Bedingungen mit feuchtem Silberoxyd behandelt, wobei eine geringe Menge desselben Alkohols resultirte.

Der Rückstand, der nach einer Reihe derartiger Bearbeitungen zurückblieb, siedete unter gewöhnlichem Druck dennoch in sehr weiten Grenzen, $130^{\circ}-200^{\circ}$, ohne sich dabei zu zersetzen. Bei der Destillation unter einem Druck von $17 \mathrm{Mm}$. geht dieser Rückstand zwischen $50^{\circ}-90^{\circ}$ über, und sobald die Temperatur sich $60^{\circ}$ genähert hatte, zeigten sich auf dem Kolbenhalse dieselben Krystalle, die schon früher erwähnt wurden. Diese Krystalle sind so flüchtig, dass sie schon beim Abpressen zwischen Filtrirpapier behufs Entfernung des flüssigen Antheils verschwinden. Die schnell durch Abpressen vom flüssigen Theile getrennten Krystalle schmolzen im zugeschmolzenen Rohr bei $\left.114^{0}{ }^{1}\right)$ Die Untersuchung des Chlorprodukts, in welchem diese krystallinische Verbindung enthalten ist, ist vorläufig aufgeschoben bis zur Herbeischaffung neuen Materials.

Bevor ich zur Untersuchung der zweiten und dritten Fraction, welche bei der Destillation der durch Zerlegung des Dibromids dargestellten Produkte erhalten wurden, übergehe, muss ich vorausschicken, dass diese Fractionen in so geringer

1) Für die Reinheit dieser Krystalle kann ich nicht garantiren, da in Folge ibrer ungewöhnlichen Flüchtigkeit von einem Umkrystallisiren nicht die Rede sein konnte. Meine Hoffnung, eine von den folgenden Dichlorverbindungen zu entdecken, hat sich nicht bestätigt:<smiles>CC(C)C(C)CCl</smiles><smiles>CC(C)C(C)CCl</smiles> 
298 Kondakow: Ueb.d.Verh.d. Tetramethylendibromidsetc.

Menge erhalten wurde, dass dieselbe nicht zur allseitigen Untersuchung hinreichte. Nichtsdestoweniger glaube ich nicht fehlzugehen, wenn ich aus den erhaltenen Daten den Schluss ziehe, dass die zweite Fraction ein Gemisch aus dem Kohlenwasserstoff der ersten Fraction und einem Aether, der in der dritten Fraction enthalten ist, darstellt. Diese letztere Fraction besteht hauptsächlich aus einem ungesättigten Aether mit dem Siedep. $130^{\circ}-134^{\circ}$. Dieser Aether, der durch seine Eigenschaften sehr lebhaft an den Aethylvaleryläther erinnert, ist keine reine Substanz, da in $\mathrm{ihm}$ eine geringe Menge eines bromhaltigen Produktes enthalten ist, welches zu entfernen in Folge des unzureichenden Materials unmöglich war.

Zur Untersuchung der krystallinischen Verbindung, welche beim Destilliren des Zersetzungsprodukts des Dibromids erhalten wurde, übergehend will ich bemerken, dass auch sie augenscheinlich aus zwei verschiedenen Substanzen besteht; wenigstens lässt ihre Krystallform darauf schliessen. Die ungereinigten Krystalle schmelzen bei $152^{\circ}$. Die Brombestimmung im ungereinigten Produkte ergab:

0,2068 (rrm. Substanz gaben 0,2627 Grm. AgBr $=54,01 \%$ Br.

Der Schmelzpunkt der Krystalle fiel nach dem Umkrystallisiren aus einer Mischung von Aether und Alkohol auf $150^{\circ}$. Sie scheiden sich aus der angegebenen Lösung in zwei Formen aus: in Form nadelförmiger Krystalle und in Form kurzer Prismen. Die letzteren scheiden sich nur in sehr geringem Maasse aus. Diese krystallinische Substanz ist sehr flüchtig und zerlegt sich sehr leicht beim Aufbewahren im Gläschen mit eingeschliffenem Stöpsel. Die weitere Untersuchung der Krystalle ist im Gange.

Auf die Beurtheilung der angeführten Daten eingehend, müssen wir zunächst feststellen, dass bei der Zerlegung des Dibromtetramethyläthylens vermittelst alkoholischer Kalilauge nicht ein Produkt, sondern mehrere erhalten werden, wobei aber der Kohlenwasserstoff der ersten Fraction vorherrscht. Dieser letztere, von Couturier als reines Tetramethyläthylen angesehene Kohlenwasserstoff besteht aus einem Gemisch von mindestens zwei Kohlenwasserstoffen, der eine von ihnen ist Tetramethyläthylen, der andere entweder Diisopropenyl oder 
Kondak ow : Ueb.d.Verh. d. Tetramethylendibromidsetc. 299 ein Kohlenwasserstoff mit geschlossener Kette. Zu diesem Schluss gelange ich, indem ich den Siedepunkt, das spec. Gew., die Eigenschaft sich zu polymerisiren ${ }^{1}$ ) und hauptsächlich sein Verhalten zu Brom und zur Chlorwasserstoffsäure in Betracht ziehe.

Zweitens muss ich bestätigen, dass die Bildung von Tetramethyläthylen, welche zuerst von Couturier constatirt wurde, keinem Zweifel unterliegt, obschon die Erklärung, die er dieser Thatsache giebt, sich nicht mit der allgemeinen Vorstellung von der Einwirkung alkoholischer Kalilauge auf die Halogenhydrine der verschiedenatomigen Alkohole vereinbaren lässt. Die von mir bei der Controlle der Couturier'schen Untersuchungen erhaltenen Daten geben noch mehr Anlass, an der Richtigkeit der Couturier'schen Erklärung zu zweifeln, und führen eher auf die Vermuthung, dass wir es hier mit

1) Das Diisopropenyldivinyl polymerisirt sich nach Couturier (S. 498) sehr leicht. Die Polymerisation der Kohlenwasserstoffe der Divinylreihe wird in meinem Laboratorium von H. Lutschinin untersucht.

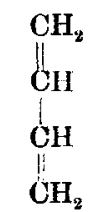

Divinyl

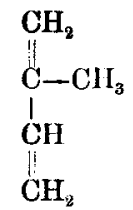

Isopren<smiles>CC(C)(C)C</smiles>

Diisopropenyl

Ob das Divinyl selbst sich polymerisiren lässt, ist bis jetat unbekannt. Das Isopren polymerisirt sich zu Dipenten I), und das dem Isopren so ähnliche Diisopropenyl müsste bei der Polymerisation Dimethyldipenten II) geben.<smiles>CC1CCC(C(C)C)CC1</smiles><smiles>CC1=CCC(C)(C(C)C)CC1C</smiles>

Die Polymerisation besteht wahrscheinlich in einer einfachen Anlagerung eines Moleküls des Kohlenwasserstoffs zum anderen. 
300 Kondakow: Ueb.d.Verh. d. Tetramethylendibromids etc.

einem neuen Fall der Isomerisation zu thun haben. Wenn diese Vermuthung sich bewahrheiten sollte, so wird es richtig sein, die Bildung des Tetramethyläthylens durch Einwirkung von alkoholischer Kalilauge auf Dibromtetramethyläthylen so zu erklären, dass das Dibromid ${ }^{1}$ ) zunächst normal zerlegt wird, analog den anderen Dibromiden in ein Monobromid mit doppelter Bindung oder geschlossener Gruppirung:

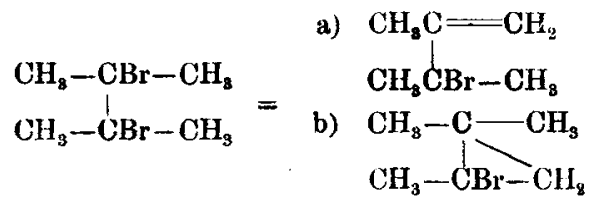

Diese Monobromide müssen unter Verlust von $1 \mathrm{Mol}$. $\mathrm{HBr}$ entweder in Diisopropenyl oder in einen der Trimethylenkohlenwasserstoffe übergehen, nach folgendem Schema:

a)

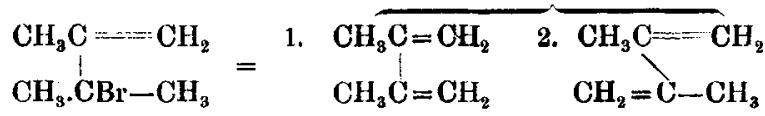

3. $\mathrm{CH}_{2} \cdot \mathrm{C}=\mathrm{CH}_{2}$ $\mathrm{CH}_{8} \mathrm{C}-\mathrm{CH}_{3}$

b) $\begin{array}{lll}\mathrm{CH}_{3}-\mathrm{C}-\mathrm{CH}_{3} \\ \mathrm{CH}_{3}-\mathrm{CBr}-\mathrm{CH}_{2}\end{array}=\begin{array}{lll}\mathrm{CH}_{3}-\mathrm{C}-\mathrm{CH}_{3} & \text { 2. } & \mathrm{CH}_{8}-\mathrm{C}-\mathrm{CH}_{3} \\ \mathrm{CH}_{3}-\mathrm{C}=\mathrm{CH} & \mathrm{CH}_{2}=\mathrm{C}-\mathrm{CH}_{2}\end{array}$<smiles>CC1(C)CC1(C)C</smiles>

Welcher von diesen Kohlenwasserstoffen dem Tetramethyläthylen beigemengt ist, konnte bis jetzt noch nicht mit Sicherheit nachgewiesen werden, obgleich es keinem Zweifel unterliegt, dass dieser Kohlenwasserstoff 2 Mol. Chlorwasserstoff und 2 Atome Brom addirt.

Doch wie, fragt es sich, soll man sich die Bildung des Tetramethyläthylens bei der in Rede stehenden Reaction denken, wenn eine einfache Abspaltung zweier Bromatome in

1) Das Pinakon giebt, ein Molekül Wasser abspaltend, zweifellos Dimethylisopropenylcarbinol: $\underset{\mathrm{CH}_{8}}{\mathrm{CH}_{3}}>\mathrm{C}(\mathrm{OH})-\mathrm{C}_{\mathrm{CH}_{3}}^{\mathrm{CH}_{3}}$, diees geht beim weiteren Abspalten eines Moleküls Wasser in Diisopropenyl über. 
diesem Falle zweifelhaft und unbewiesen erscheint? Ich will es vorläufig, in Anbetracht des unzureichenden factischen Materials, unterlassen, auf diese Frage eine entscheidende Antwort zu geben. Man kann vorläufig darüber blos mehr oder weniger wahrscheinliche Vermuthungen aufstellen, indem man annimmt, dass entweder die Monobromide unter den Bedingungen der Reaction sich in Tetramethyläthylen verwandeln, oder aber, dass die Kohlenwasserstoffe - Diisopropenyl und die Trimethylenkohlenwasserstoffe - sich zu diesem Hexylen isomerisiren.

In ersterem Falle würde die Reaction durch folgendes Schema veranschaulicht werden:

a)

b)

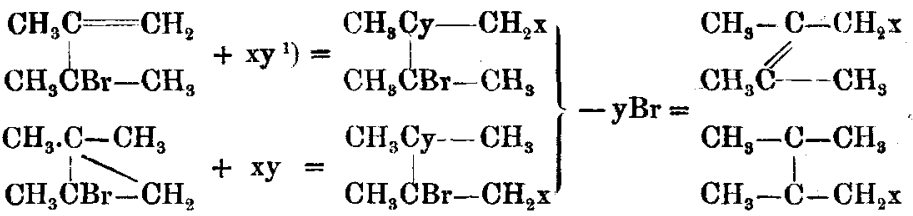

Wenn aber die Kohlenwasserstoffe (Diisopropenyl oder die trimethylenartigen) unter dem Einfluss von alkoholischer Kalilauge in Tetramethyläthylen übergehen sollten, so würde es ein neuer, sehr interessanter Fall der Isomerisation von Cykloparaffinen, Cykloolefinen und den Derivaten des Divinyls in Olefine sein, ein Fall, der eine auf Thatsachen beruhende Untersuchung erfordert.

Ich gedenke diese Voraussetzungen in Bälde auf ihre Richtigkeit zu prüfen, indem ich einerseits das Verhalten der alkoholischen Kalilauge zu verschiedenen, der Structur nach dem Tetramethyläthylendibromid analogen Dibromiden, andererseits das Verhalten der Kohlenwasserstoffe der Polymethylenund Divinylreihe zu derselben Reaction untersuchen werde.

Bei der Ausarbeitung der factischen Theile vorliegender Untersuchung ist mir $\mathbf{H}$. Levites behülflich gewesen, wofür ich ihm hiermit danke.

Die Untersuchungen werden fortgesetzt.

1./12. Januar 1899.

1) xy muss noch bestimmt werden. 